\title{
Review of inflammatory biomarkers in hospitalized adults with suspected infection
}

\author{
Mark D Lacy MD, FIDSA
}

\begin{abstract}
Quantitative measurement of inflammatory biomarkers is frequently obtained in evaluating hospitalized patients with suspected or confirmed infections and other inflammatory disorders. These markers may be used to establish a diagnosis or assess response to therapy. Consensus on which marker to use and when to use them is not settled nor to what extent these indicators improve outcomes or quality of care. The following is a brief review of the common inflammatory biomarkers and a suggested approach for their utilization.
\end{abstract}

Keywords: Inflammatory disease, infection, biomarkers, erythrocyte sedimentation rate, ESR, c-reactive protein, CRP, procalcitonin, ferritin, acute phase reactants

Medical decision making integrates a composite of clinical factors and corroborative laboratory investigations. In cases presenting with features suspicious for infection or other inflammatory disorders, the diagnostic interpretation of clinical findings may be improved by the detection of an increase in acute phase reactants. These findings may impact decisions about antibiotics in febrile critically ill patients. Acute phase reactants are serum proteins that increase with inflammation and tissue injury and include C-reactive protein (CRP), amyloid, ferritin, fibrinogen, complement alpha-2 macroglobulin, procalcitonin (PCT), and others. Interleukin-6 and tumor necrosis factor are examples of cytokines that trigger the synthesis and release of acute phase reactants (Figure). The following discussion provides a brief review of commonly available indicators of acute phase reactions and some of the syndromes in which they may be clinically useful.

One of the oldest of these tests is the erythrocyte sedimentation rate (ESR), which is an indirect

Corresponding author: Mark Lacy

Contact Information: Mark.lacy@ttuhsc.edu

DOI: 10.12746/swrccc.v6i26.501 indicator of inflammation. The ESR is affected by increasing concentrations proteins such as alpha globulins and fibrinogen. Erythrocytes are negatively charged and in the presence of acute phase reactants, these positively charged proteins increase the rate at which red blood cells settle in the plasma. The reported value reflects the distance in millimeters red blood cells descend in a tube over 1 hour. The ESR increases with age in healthy people. The test may be confounded by factors unrelated to inflammation, such as plasma albumin concentrations and the size, shape, and number of red blood cells. Elevations of ESR may occur in the presence of abnormal immunoglobulins, anemia, end-stage renal disease, obesity, and pregnancy. The inflammation associated with malignancy, diabetes, ischemia, and collagen vascular diseases tends to increase fibrinogen levels and, thereby, increase the ESR. The long half-life of fibrinogen may be one reason the ESR is slow to increase and slow to decrease to normal levels compared to other acute phase reactants, such as the CRP. While ESR is typically elevated in serious infectious, rheumatic, and neoplastic diseases, it can be normal in critically ill patients. ${ }^{1}$ The expected value can be estimated roughly by dividing the age in years by 2 in men; add 10 to age in years for 


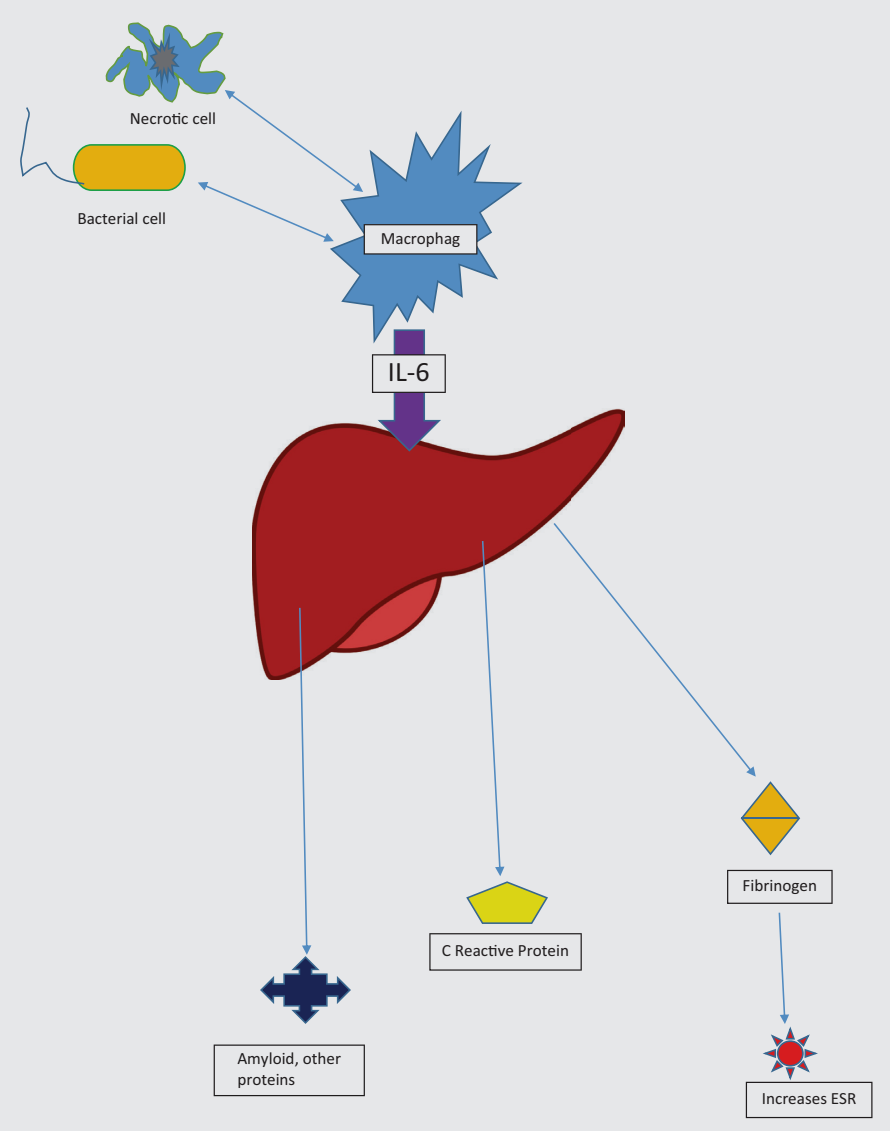

Figure. Pathways for the production of acute phase reactants.

women and divide by 2 . Factors that affect the ESR are summarized in the Table.

C-reactive protein is a component of the innate immune response produced by the hepatocytes. It binds to damaged tissue and microbes by phosphocholine, present on the cell wall of bacterial and some fungal pathogens, eliciting complement fixation and phagocytosis. Unlike ESR, CRP responds quickly to an inflammatory process whether triggered by infection, tissue necrosis, or autoimmunity. Maximum levels may be reached in less than 2 days; normalization occurs within hours or days after inflammation subsides. C-reactive protein levels trend higher in obese patients. ${ }^{2}$ Patients with liver disease and overwhelming
Table.

\begin{tabular}{|l|l|}
\hline Factors increasing ESR & Factors decreasing ESR \\
\hline Renal failure & Leukocytosis \\
\hline Pregnancy & Low fibrinogen \\
\hline Female gender & Malnutrition \\
\hline Hypercholesterolemia & Polycythemia \\
\hline $\begin{array}{l}\text { B cell tumors, } \\
\text { macroglobulinemia }\end{array}$ & $\begin{array}{l}\text { Sickle cell disease, } \\
\text { abnormal RBCs }\end{array}$ \\
\hline Aging & Congestive heart failure \\
\hline Anemia & \\
\hline
\end{tabular}

sepsis may have blunted CRP responses. ${ }^{3}$ Similar to the ESR, normal CRP levels appear to vary with age and sex. Analysis of nearly 9000 Americans over age 20 reported a mean level of $3.4 \mathrm{mg} / \mathrm{liter}$ in men (median 1.6) and a mean of $5.1 \mathrm{mg} / \mathrm{liter}$ in women (median 2.7). ${ }^{4}$ Other sources have reported the 95th percentile value of CRP in the overall population is $0.95 \mathrm{mg} / \mathrm{dl}$ for males and $1.39 \mathrm{mg} / \mathrm{dl}$ for females. A proposed calculation for CRP is the upper limit of the reference range $(\mathrm{mg} / \mathrm{dL}$ ) equals (age in years)/50 for men and (age in years $/ 50)+0.6$ for women. ${ }^{5}$

There are a couple caveats related to CRP values to consider. Occasionally, one encounters highsensitivity CRP nomenclature as if this parameter differs from standard CRP measurement. In fact, there is no difference between the two, and high-sensitivity merely reflects CRP measurement using an assay that can detect very low levels of the protein. The CRP that is measured by this method differs in no structural or physiologic way from that measured by less sensitive methods. Second, diagnosticians need to be aware there is variation in the units by which CRP levels are reported (evident in the comments above) with some laboratories measuring CRP concentrations in $\mathrm{mg} / \mathrm{dL}$, others using $\mathrm{mg} / \mathrm{L}$, with high-sensitivity and cardiology-oriented assays typically reporting the latter units.

Sometimes discrepant results are found between ESR and CRP measurements, and CPR is generally 
considered a more sensitive marker of acute phase reactions. ${ }^{6}$ Patients with high CRP but normal ESR typically have infection, ischemia, or thromboembolism. ${ }^{7}$ Patients with high ESR but normal CRP may have systemic inflammatory or autoimmune processes, including those associated with malignancy. ${ }^{8}$ For example, patients with very active systemic lupus erythematosus may have normal CRP even with an elevated ESR; this could be related to high levels of type 1 interferon that can inhibit hepatic production of CRP.

Procalcitonin is a pre-hormone produced by thyroid $C$ cells but in sepsis can be produced in extrathyroidal tissues. Its role in inflammation, including that stimulated by bacterial and some fungal infections, is not completely understood. Serum concentrations wax and wane with the severity of the infectious process. The substance is down regulated with viral infections. Levels increase within 6-12 hours of bacterial infection when triggered by release of proinflammatory mediators. Procalcitonin appears to be more specific for bacterial infections than C-reactive protein or white blood cell counts. ${ }^{9}$ Further, PCT concentrations decline quickly during resolution of infection and may be useful for following responses to therapy. However, PCT can be elevated in many syndromes associated with marked inflammation, such as severe burns, extensive trauma, or acute pancreatitis. Although PCT is a potentially useful diagnostic test, the use of PCT levels varies widely among hospitals probably because clinicians are not universally convinced that it is reliable. ${ }^{10}$

Ferritin is a ubiquitous protein known for its role in iron storage and is present in intra-cellular compartments, such as macrophages, and to a lesser extent in serum. Regulation of this protein is controlled by both iron stores and various cytokines and hormones. Low serum ferritin is often used to corroborate iron deficiency. Hyperferritinemia is recognized in the same inflammatory states that trigger elevations of other acute phase reactants, but levels usually don't increase over 2 to 3 times normal in these disorders. Liver disease due to viral hepatitis, alcoholic hepatitis, or nonalcoholic steatohepatitis can elevate serum ferritin despite normal body iron stores. Hyperferritinemia may occur in iron overload states, such hemochromatosis, hemophagocytic lymphohistiocytosis, and hemolytic disorders.

\section{Clinical utility and applications}

Mild or moderate increases in acute phase reactants are rarely helpful in establishing a differential diagnosis. Greater utility may be found with multifold increases in these parameters. On occasion, moderate increases in acute phase reactants can reinforce a clinical impression or can be used to assess response to therapy. The following discussion highlights some clinical scenarios in which the measurement of acute phase reactions can be helpful in hospitalized patients.

\section{SEPSIS}

Management of patients who present with sepsis or sepsis-like illness is commonly a dilemma. Physicians know that a million patients are affected by bacterial sepsis every year in the United States and appropriate, rapid initiation of antibiotics correlates with enhanced survival. Conversely, delayed or inappropriate empiric therapy correlates with worse outcomes ${ }^{11}$ including those related to indiscriminate and prolonged use of antimicrobials, drug resistance and increasing the risk of adverse drug events. Tools to facilitate this decision making are desirable, but ESR and CRP measurements are too non-specific to elucidate sepsis syndromes with confidence.

Randomized controlled studies evaluating PCT performance in the ICU are sparse, and those which exist are not robust. Many studies evaluating PCT at the bedside of the septic patient have been difficult to compare because different PCT cutoff concentrations have been used in randomized trials. Layios et al compared over 251 ICU patients in a control group and 258 patients in a PCT-guided therapy group. Patients in the latter group had no reduction in antibiotic exposure, and PCT measurements were not highly accurate in differentiating infected/probably infected patients from unlikely infected patients. 
This was determined following a posteriori review of cases by infectious disease specialists blinded to the PCT levels. ${ }^{12} \mathrm{~A}$ meta-analysis assessing PCT obtained early in the ICU admission of systemic inflammatory response syndrome patients who were not immunocompromised was published in 2006. The PCT sensitivities and specificities in sepsis from 25 studies varied widely. Procalcitonin was more accurate in detecting sepsis cases than CRP with odds ratio of an elevated PCT in infected patients $>15$ compared to uninfected patients. ${ }^{13}$ Based on this, elevated PCT may be a helpful correlate in the diagnosis of sepsis and a prediction of organ dysfunction.

Hemophagocytic lymphohistiocytosis $(\mathrm{HLH})$ is a rare sepsis mimic, affecting perhaps 1 in 800,000 persons yearly. It usually presents as a febrile syndrome with multiorgan dysfunction. Its pathophysiology is rooted in activated macrophages, which fail to respond to down-regulation by natural killer (NK) cells and cytotoxic T lymphocytes. Cytokine storm and hemophagocytosis by macrophages follow. While primary HLH is a rare disease of childhood, secondary HLH can occur in adults. The most common triggers include various pathogens, such as HIV, herpes viruses, influenza, hepatitis $C$ virus, rickettsia, Mycobacterium tuberculosis, and possibly Gram-negative aerobes. Other associated triggers include lymphomas and other malignancies, rheumatologic disorders, and immunodeficiency states. A sine qua non of this diagnosis is extreme hyperferritinemia with levels frequently exceeding $10,000 \mathrm{ng} / \mathrm{mL} .^{14}$ There are few disorders other than $\mathrm{HLH}$ which result in extreme hyperferritinemia.

\section{Pneumonia}

Acute respiratory tract infections are a major indicator for adult hospitalization and death worldwide and comprise a primary cause of antibiotics overuse. While more than $40 \%$ of respiratory infections have a viral and not a bacterial cause, current diagnostic platforms lack the precision to guide therapy, at least initially. Several meta-analyses in the last decade have suggested that the PCT may be a tool to reduce antibiotic exposure, but its effect on clinical outcomes as mentioned above with sepsis has not been conclusive. A meta-analysis published in 2017 of patients with community-acquired pneumonia, hospital-acquired pneumonia, ventilator-associate pneumonia, and exacerbation of COPD explored whether PCT measurements affected all-cause mortality at 30 days and antibiotic use. There was a significant decrease in adverse effects from antibiotics, a 2.4-day reduction in antibiotic exposure in PCT guided patients, and a $18 \%$ reduction in mortality compared to controls in whom PCT did not guide therapy. The length of stay was the same in both groups. ${ }^{15}$ The Procalcitonin Antibiotic Consensus Trial (ProACT) studied 1,656 emergency department patients from 14 hospitals with suspected lower respiratory tract infection randomized to a PCT guided intervention compared to usual care. Primary outcome was antibiotic utilization and adverse events attributable to withholding antibiotics. The provision of PCT levels during the clinical encounter did not reduce antibiotic utilization nor did it lead to worse outcomes. Thus a PCT-guided algorithm did not result in increased adverse events in patients. ${ }^{16}$ Part of this may be related to the lower acuity of patients with lower PCT levels, the latter providing only modest additional data to guide decision making at the bedside.

\section{Musculoskeletal INFECTION}

Elevated ESR and CRP are non-specific and do not increase pre-test likelihood of septic arthritis. ${ }^{17} \mathrm{~A}$ retrospective chart review of over 160 patients with acute arthritis, 44 of whom had septic joints, indicated that the ESR was not predictive of septic joints and that a normal CRP could not be used to exclude infection. ${ }^{18}$ Procalcitonin is more sensitive and specific than ESR and CRP based on meta-analysis of patients with fever and joint symptoms and signs and can support a suspected diagnosis. However, a normal PCT does not exclude a septic joint and can be elevated after trauma or immediately after surgery. ${ }^{19}$

In patients with acute osteomyelitis, the ESR can be used to assess response to therapy provided co-morbidities and other factors (see above) don't confuse its interpretation. In chronic osteomyelitis, by 
definition no longer an "acute phase" infection, ESR and CPR can be normal and do not reliably exclude the diagnosis and are rarely helpful in management. If spinal infection or discitis is suspected, the same cautions apply due to lack of high sensitivity and specificity though measurements can help guide the duration of therapy for these conditions.

\section{ACUTE BACTERIAL SKIN INFECTIONS}

Cellulitis, fasciitis, and other acute bacterial skin infections are among the most common infectious disease syndromes resulting in hospitalization, occasionally requiring critical care resources. Acute phase reactants might serve as an adjunct in the clinical evaluation, but there are few studies in patients with these infections. Most patients with significant acute bacterial skin infections will have elevated CRP, PCT, and white blood counts. One recent small study demonstrated acute phase reactants levels tended to be higher in patients requiring hospitalization, were generally higher in patients with abscesses, and tended to decline by day 3 to 5 of successful therapy. Acute phase reactants measurements may help in decision making related to duration of antibiotic therapy in these cases. ${ }^{20}$

\section{SUMmARY}

Acute phase reactants have a supplementary role in the management of hospitalized patients with fever. The ESR and CRP, while not specific enough to establish a diagnosis, can be helpful in guiding the intensity and duration of anti-infective therapy. With a compatible clinical scenario, extremely elevated ferritin levels may suggest HLH. Procalcitonin may have some utility in determining whether a febrile illness is due to bacterial or non-bacterial pathogens, particularly in patients with pneumonia. Procalcitonin may have a role in the de-escalation of antibiotic and reducing antibiotic overuse. While not perfect tools, acute phase reactants have some role in formulating a diagnostic and therapeutic plan and may improve antimicrobial stewardship.
Article citation: Lacy MD. Inflammatory biomarkers in hospitalized adults with suspected infection. The Southwest Respiratory and Critical Care Chronicles 2018;6(26):4-9

From: Department of Internal Medicine at Texas Tech University Health Sciences Center in Lubbock, TX

Submitted: $1 / 3 / 2018$

Accepted: $7 / 27 / 2018$

Reviewers: Victor Test MD, David Griffith MD

Conflicts of interest: none

This work is licensed under a Creative Commons

Attribution-ShareAlike 4.0 International License

\section{REFERENCES}

1. Bitik B, Mercan R, Tufan A, et al. Differential diagnosis of elevated erythrocyte sedimentation rate and C-reactive protein levels: a rheumatology perspective. Eur J Rheumatol 2015;4:131-4.

2. Visser M, Bouter LM, McQuillan GM, et al. Elevated C-reactive protein levels in overweight and obese adults. JAMA 1999;282(22):2131-2135.

3. Silvestre J, Coelho L, Povoa P. Impact of fulminant hepatic failure in C-reactive protein. J Crit Care 2010;25:e7-12.

4. Woloshin S, Schwartz L. Distribution of C-reactive protein values in the United States. N Engl J Med 352;15:1611-1613.

5. Wenner M, Daum P, McQuillan G. The influence of age, sex, and race on the upper reference limit of serum C-reactive protein concentration. J Rheumatol 2000;27(10):2351.

6. Kushner I. Acute phase reactants. Up-to-date 2015 Jul 15.

7. Felman M, Aziz B, Kang G, et al. C-reactive protein and erythrocyte sedimentation rate discordance: frequency and causes in adults. Translational Research 2013;161:37-43.

8. Harrison M. Australian Prescriber 2015;38:93-4.

9. Christ-Crain M, Muller B. Biomarkers in respiratory tract infections: diagnostic guides to antibiotic prescription, prognostic markers and mediators. Eur Respir J 2007;30:556-73.

10. Chu D, Mehta A, Walkey A. Practice Patterns and Outcomes Associated with Procalcitonin Use in Critically Ill patients with Sepsis. Clin Inf Dis 2017;64:1509-1514.

11. Eagye K, Kuti J, Nicolau D. Risk factors and outcomes associated with isolation of meropenem high-level-resistant Pseudomonas aeruginosa. Infect Control Hosp Epidemiol 2009;30:746-752.

12. Layios N, Lambermont B, Canivet J, et al. Procalcitonin usefulness for the initiation of antibiotic treatment in intensive care unit patients. Crit Care Med 2012;40(8):2304-2309. 
13. Uzzan B, Cohen R, Nocolas $P$, et al. Procalcitonin as a diagnostic test for sepsis in critically ill adults after surgery or trauma: A systematic review and meta-analysis. Crit Care Med 2006;34:1996-2003.

14. Grange S, Buchonnet G, Besneir E, et al. The use of ferritin to identify critically ill patients with secondary hemophagocytic lymphohistiocytosis. Crit Care Med 2016; 44(11):e1045-1053.

15. Schuetz P, Wirz Y, Sager R, et al. Effect of procalcitoninguided antibiotic treatment on mortality in acute respiratory infections: a patient level meta-analysis. Lancet/Infection 2017; 17:1-13.

16. Huang D, Yealy D, Filbin M, et al. Procalcitonin-guided use of antibiotics for lower respiratory infection. NEJM 2018; 379:236-49.
17. Matthews CK, Weston VC, Jones A, et al. Bacterial septic arthritis in adults. Lancet 2010;375:846-855.

18. Ernst A, Weiss S, Trach L, et al. Usefulness of CRP and ESR in predicting septic joints. South Med Journal 2010; 103:522-526.

19. Shen CJ, Wu MS, Lin $\mathrm{KH}$, et al. The use of procalcitonin in the diagnosis of bone and joint infection: a systemic review and meta-analysis. Eur J Clin Microbiol Infect Dis 2013; 32:807-814.

20. Jenkins $\mathrm{T}$, Haukoos $\mathrm{J}$, Cotton $\mathrm{E}$, et al. Time course of C-reactive protein and procalcitonin levels during the treatment of acute bacterial skin infections. OFID; Downloaded from https://academic.oup.com/ofid/article. 03 April 2018. 